\section{Development and Clinical Application of Percutaneous Compressive Locking Intra- medullary Nail for Humerus}

\author{
Zhang Zuojun*, Niu Zuling, Chang Zhongxiao, Zhang Chuan, \\ Zhang Di
}

Orthopedic Institute of Henan Province and Luoyang Orthopedic Hospital of Henan Province

\begin{abstract}
Objective: To simplify the usage of locking intramedullary nail; to increase the therapeutic effect and decrease the medical costs for patients.

Methods: According to the proximal constitution of the humerus and the anatomic features of the medullary cavity, percutaneous compressive locking intramedullary nail was developed. Mechanical tests and clinical observation were performed. All the data were compared and analyzed, such as firmness of fixation, result of reduction, fracture union, joint function recovery, the simplicity of application and medical costs, etc.

Results: Through mechanical tests, it is proved that all the indices of percutaneous compressive locking intramedullary nail can meet the requirements of biomechanical fixation of humeral fractures. In addition, clinical observation of 1078 cases further proved that percutaneous compressive locking intramedullary nailing for humeral fractures has the advantages of easy application, firm fixation, fast fracture union, early function recovery and so on.

Conclusion: Percutaneous compressive locking intramedullary nailing is a simple and reliable method for humeral fracture. It can be spread and widely used.
\end{abstract}

Keywords: Humeral fracture; Locking intramedullary nail; Mechanical test

\section{Introduction}

Humerus fracture is a common kind of fracture which account for $3-5 \%$ of all fractures [1]. Most humerus fractures can be treated by closed reduction and splinting. However, some unstable fractures often require open reduction and internal fixation. Major internal fixation use metal plates and intramedullary fixation nail (pin). Intramedullary fixation makes no destruction to external periosteum, has faster healing, less stress shielding, fewer refracture after

*Corresponding author: Zhang Zuojun, Orthopedic Institute of Henan Province and Luoyang Orthopedic Hospital of Henan Province, No 82 South Qiming road, Luoyang, Henan, China, Tel: +86 13838838000, +86 37963546746; Fax: +86 37963552102; E-mail: zzjlip@163.com

Citation: Zuojun Z (2015) Development and Clinical Application of Percutaneous Compressive Locking Intramedullary Nail for Humerus. J Orthop Res Physiother 1: 009.

Received: May 21, 2015; Accepted: June 20, 2015; Published: August 20, 2015 removal and larger fixing force, and has no need for external fixation [2]. Therefore, intramedullary fixation is used more and more in clinical practice [3]. However, intramedullary nails currently used are complicated in structure, cumbersome to operate, and require open reduction, open nailing and second operation for removal. This brings great pain and large cost to patients. Additionally, intramedullary nails currently used lack compression at the fracture sites, likely leading to delayed healing or even no healing. In view of this, we carefully studied the anatomical characteristics of humerus, and developed the Simple Percutaneous Compressive Locking Intramedullary Nail (SPCLIN). We have done 1078 clinical trials, and got good results, which are summarized in this study.

\section{Introduction of instruments}

SPCLIN is made by the internationally standard stainless steel 317L. It has two main parts, namely, an intramedullary nail and a locking nail. Intramedullary nail is cylindrical with distal $1 / 4$ part as cancellous bone screw, which is used to rotate the nail forward. The tail of intramedullary nail is swelling into a spherical knob, in contact with the greater tuberosity of humerus after the nail is screwed in. This structure brings axial compression, and leads to intercalation of fracture sites. The spherical knob of the tail has a groove, which is used for increasing the pressure of spiral knife. A $45^{\circ}$ slant-hole close to the groove is made to facilitate the sliding of locking nail. Locking nail is the $3.5 \mathrm{~mm}$ cancellous bone screw that is for general medical use. The tail has hexagonal notch that is used for screwing the locking nail (Figure 1).

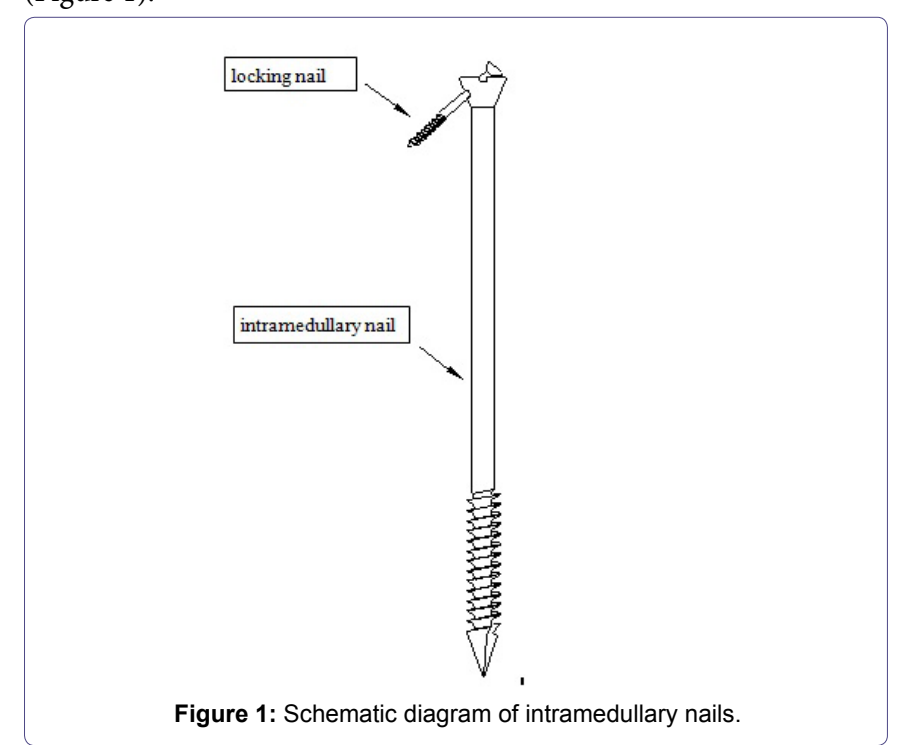

\section{Mechanical Test}

\section{Mechanical test of percutaneous compressive locking intramedullary nail (bare nail test)}

Test four $6.5 \mathrm{~mm} \times 270 \mathrm{~mm}$ SPCLINs under universal material testing machine Shimadzu AGS10KNG. We have done vertical compression test, three-point bending test and load to failure test of locked structure. 
Compression test: Screw the threaded intramedullary nail into the special loading base to have the compression test. The compressive modulus was $5213 \mathrm{mN} / \mathrm{mm}^{2}$ with strength greater than $339 \mathrm{~N} / \mathrm{mm}$.

The compressive modulus was $5213 \mathrm{mN} / \mathrm{mm}^{2}$ with strength greater than $339 \mathrm{~N} / \mathrm{mm}$.

Bending test: Place the intramedullary nail horizontally in the testing machine so as that spacing between two bending points is $80 \mathrm{~mm}$. Force was loaded in the middle of the nail. The bending strength was $1631 \mathrm{~N} / \mathrm{mm}^{2}$; and the modulus of elasticity was $119287 \mathrm{~N} / \mathrm{mm}^{2}$.

Bending test of locking nail: spacing between two bending points was $20 \mathrm{~mm}$. Bending strength of the nail body was $1203 \mathrm{~N} / \mathrm{mm}^{2}$; and flexible modulus was $61886 \mathrm{~N} / \mathrm{mm}^{2}$.

Damage test of locked structure: Intramedullary nail was locked with locking nail. Force was $83.6 \mathrm{~N}$ pre-load, then continued to $495 \mathrm{~N}$ until the pressure load begun to decrease and the body of locking nail begun to bend. The body of locking nail broke when $2627 \mathrm{~N}$ was loaded. Proximal locking notch of intramedullary nail showed no significant change when nail body was broken. All mechanical tests showed that mechanical properties of various components of SPCLIN can meet the need of fixation.

\section{Comparing the installation time between SPCLIN and traditional intramedullary nail}

18 adult humerus specimen (provided by Shanghai University of Traditional Chinese Medicine Department of Anatomy) were bent in the middle and fastly broken with a mechanical testing machine, resulting in the model of middle fracture of humerus. Experimental group was fixed using SPCLIN according to method one of internal fixation. Control group was fixed using traditional intramedullary nail according to method two of internal fixation. Internal fixation was operated by two most skillful doctors whom are all chief doctor (They have experienced more than 1000 operations of humerus fracture). Time used were recorded and compared.

Method one of internal fixation: A $5 \mathrm{~mm}$ diameter hole is opened at the humeral greater tuberosity at the direction toward the marrow cavity. Intramedullary nail is thwarted into the hole, and hammered to make it completely into the bone marrow cavity. Fracture sites were then reduced; and the intramedullary nail is screwed into the distal end of the humerus bone to make the tail at the same height of greater tuberosity. Adjust the direction of nail hole towards the inside-down of anatomical neck of humerus, and use screwdriver to nail the locking nail into the hole (Figure 2).

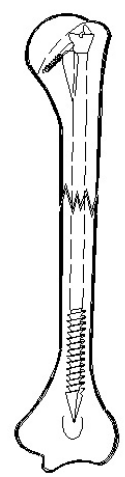

Figure 2: Schematic diagram of fixation.
Method two of internal fixation: A $5 \mathrm{~mm}$ diameter hole is made same as above. Humerus marrow cavity is then expanded to $7.5 \mathrm{~mm}$ with depth meeting the need of intramedullary nail installation. Put the 7mm G-K intramedullary nail on the remote target locator. Then put the nail into the expanded bone marrow cavity to reduce the fracture sites. With the help of remote target locator, drill the distal nail hole and nail the locking nail. Install the proximal nail target locator. With the help of proximal target locator, drill the proximal locking hole. Reduce accurately the fracture; and screw firmly the proximal locking nail. Remove the target locator.

The results showed that installation time for SPCLIN is about 3 minutes and installation time for traditional nails is about 7 minutes. About $43 \%$ of installation time is saved by using SPCLIN (Table 1).

\begin{tabular}{|c|c|c|c|c|c|c|c|}
\hline \multirow{2}{*}{ Group } & \multicolumn{5}{|c|}{ Installation time of internal fixation } & Total \\
\cline { 2 - 8 } & $\mathbf{1}$ & $\mathbf{2}$ & $\mathbf{3}$ & $\mathbf{4}$ & $\mathbf{5}$ & $\mathbf{6}$ & \\
\hline $\begin{array}{c}\text { Experimental } \\
\text { group (SPCLIN) }\end{array}$ & $3^{\prime} 24^{\prime \prime}$ & $\mathbf{2}^{\prime} 09^{\prime \prime}$ & $\mathbf{2}^{\prime} 43^{\prime \prime}$ & $\mathbf{2}^{\prime} 48^{\prime \prime}$ & $3^{\prime} 31^{\prime \prime}$ & $\mathbf{2}^{\prime} 566^{\prime \prime}$ & $2^{\prime} 55^{\prime \prime} \pm 0^{\prime} 30^{\prime \prime}$ * \\
\hline $\begin{array}{c}\text { Control group } \\
\text { (traditional } \\
\text { intramedullary } \\
\text { nail) }\end{array}$ & $6^{\prime} 41^{\prime \prime}$ & $6^{\prime} 51^{\prime \prime}$ & $10^{\prime} 40^{\prime \prime}$ & $7^{\prime} 49^{\prime \prime}$ & $7^{\prime} 02^{\prime \prime}$ & $6^{\prime} 48^{\prime \prime}$ & $7^{\prime} 39^{\prime \prime} \pm 1^{\prime} 32^{\prime \prime}$ \\
\hline Time difference & $3^{\prime} 17^{\prime \prime}$ & $4^{\prime} 42^{\prime \prime}$ & $7^{\prime} 57^{\prime \prime}$ & $5^{\prime} 01^{\prime \prime}$ & $3^{\prime} 31^{\prime \prime}$ & $3^{\prime} 52^{\prime \prime}$ & $4^{\prime} 33^{\prime \prime} \pm 1^{\prime} 43^{\prime \prime}$ * \\
\hline
\end{tabular}

Table 1: Comparison of in vitro installation time between SPCLIN and traditional intramedullary nail.

Note: $p<0.001$

\section{Comparison of mechanical properties of fixation between SPCLIN and traditional intramedullary nail}

6 pairs of SPCLIN-fixed humerus fracture models and 6 pairs of traditional nail-fixed ones were tested in the mechanical testing machine for compression test, bending test and torsional test.

Compression test: Loading speed was $5 \mathrm{~mm} / \mathrm{min}$. Test results are shown in figure 1 . The results showed that: (1) when compressive displacement is between 0.5 and $3.5 \mathrm{~mm}$, SPCLIN-fixed group has higher compressive load than control group; (2) when compressive displacement is between 4 and $4.5 \mathrm{~mm}$, control group has higher compressive load than SPCLIN-fixed group; and (3) only when compressive displacement is at 0.5 and $1 \mathrm{~mm}$, difference of compressive load is significant $(\mathrm{P}<0.05)$.

Bending test: Three points bending test was conducted. Span was $180 \mathrm{~mm}$. Loading speed was $5 \mathrm{~mm} / \mathrm{min}$. Data is shown in figure 2 . The results show that the average bending moment of SPCLIN-fixed group is not less than control group for each displacement. However, the difference between two groups for each displacement is not significant. This indicates that anti-bending property between two groups has no significant difference.

Torsional test: After the intramedullary nail is fixed, same square blocks were cast at the ends of humerus specimens with denture powder. Testing machine then clasped at the square blocks. Clockwise torsion was loaded with speed of $5 \%$ min (Figure 3 ).

Test results showed that: (1) between $0.25^{\circ}$ and $1.50^{\circ}$ twist angle, SPCLIN has greater torque than control group; (2) between $2.0^{\circ}$ and $5.00^{\circ}$ twist angle, control group has greater torque than SPCLIN group. The difference is significant only at $0.25^{\circ}$ and $0.50^{\circ}(\mathrm{P}<0.05)$.

From above data, we can easily see that SPCLIN and traditional nails have no significant difference in fixation results and performance, and can both meet the need of fracture fixation. 


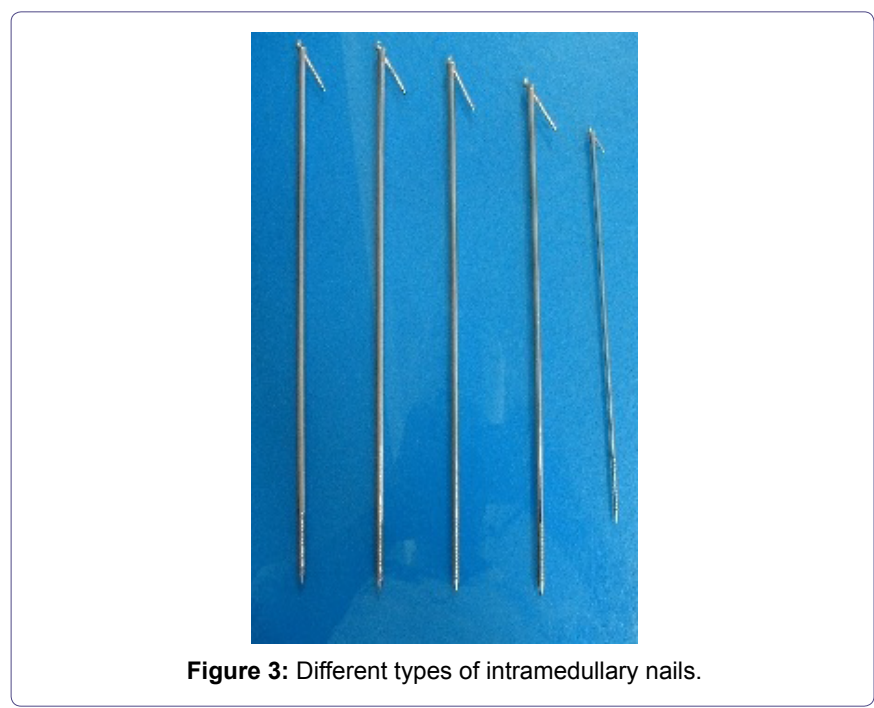

\section{Clinical Observation}

\section{General information}

This retrospective study was carried out at department of orthopedic surgery in Luoyang orthopedic hospital, Traditional Chinese medicine hospital of Henan province and The $2^{\text {nd }}$ Traditional Chinese medicine hospital of Luoyang from January 2009 to November 2012. The work was approved by institutional medical ethics committee. 617 cases were male; and 461 cases were female. 558 cases were of the left side; 520 cases were of the right side. Ages of patients ranged from 18 to 78 years old with mean age of 47.5 years. 72 cases had concurrent ipsilateral Colles fractures. 34 cases had ipsilateral clavicular factures. 23 cases has ipsilateral rib fractures. 33 cases had radial nerve injury. 11 cases had brachial plexus injury. All patients were of blunt trauma. The longest treatment was 19 days; and shortest was 2 hours. Average was 9.6 days. 541 cases had transverse fracture. 353 cases had oblique fracture. 184 cases had comminuted fracture.

\section{Treatment procedure}

Patients laid with supine position. Shoulders were padded to elevate $10 \mathrm{~cm}$ height. Take left humerus fracture for example: after anesthesia becomes effective, do the routine disinfection. Left upper extremity is then internally rotated $15^{\circ}$. Under X-ray monitoring, put the nail in line with the skin to determine the length of the intramedullary nail. Nail tail should be at the same height with greater tuberosity; and nail tip should be of $0.5 \mathrm{~cm}$ longer than the olecranon fossa. After suitable intramedullary nail is selected, at $0.5 \mathrm{~cm}$ before the tip of the acromion, prick a small skin incision of about $0.5 \mathrm{~cm}$. With the opening device, open a $5-8 \mathrm{~mm}$ bone hole in the greater tuberosity of humerus in the direction toward the bone marrow cavity (depending on the thickness of nails). Nail the tip of nail into the hole. Hammer the nail until the nail tip arrives at the fracture site. Reduce the fracture at this time. After reduction, continue to hit the nail till nailing through the fracture site. When exposed nail tail is about $5-8 \mathrm{~cm}$ (depending on different lengths of nails), switch to use T-screw to rotate the nail completely into the skin with tail at the same height as greater tuberosity. Adjust the direction of hole for locking nail toward the inside-down of anatomical neck of humerus. Screw the locking nail into the locking nail hole. Rotate the limb to make sure that fixation is stable and both intramedullary and locking nail have no loosening (Figures 4 and 5). Wrap the nail holes. Use antibiotics routinely for a week. Splinting is made over the shoulder and over the humeral elbow.
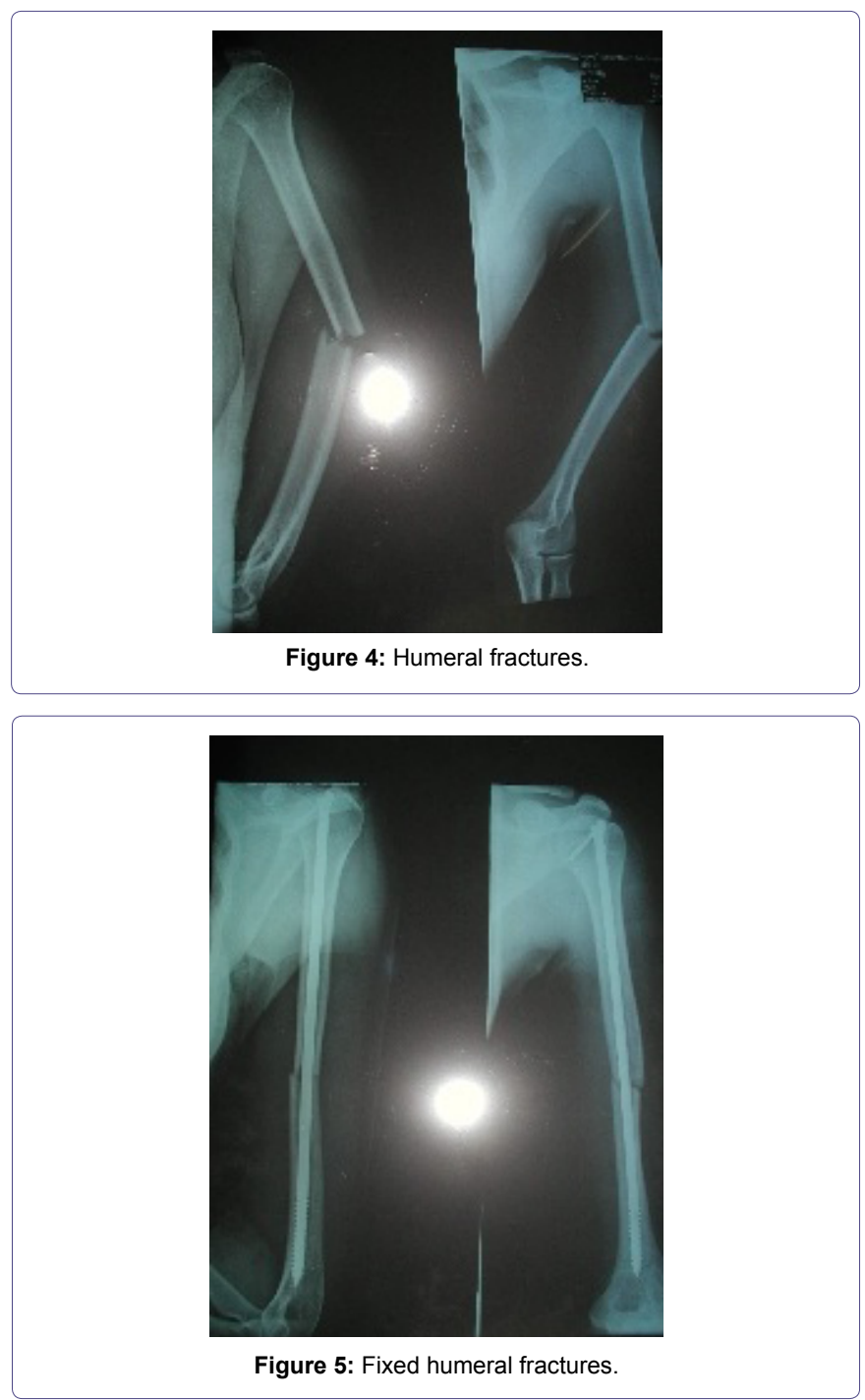

\section{Results of treatment}

1006 out of 1078 cases had six months follow-up. 11 elderly cases died after discharge. 72 cases were lost in follow-up. 113 out of the 1006 followed cases had $100 \%$ matching. Healing rate was $75.1 \%$ at 2 months follow-up. Shoulder recovery rate was $85.9 \%$ by UCLA scoring method [4]. At 3-month follow-up, clinical fracture healing rate was $89.7 \%$; and functional recovery was $97.2 \%$. At six months follow-up, bone fracture healing was $100 \%$; and functional recovery was $99.1 \%$. There was no single case of infection, broken nail, lagged nail, bent nail or unhealed fracture.

\section{Discussion}

\section{Selection of intramedullary nail}

Humerus's marrow cavity was irregular in shape. Marrow cavity starts at about 10/30-11/30 of humerus, and ends at 26/30-28/30 [5]. At sectional view, marrow cavity is round-shaped with outer layer of compact bone and inner layer of cancellous bone. Inside cancellous bone lies the pure marrow cavity. Effective marrow cavity includes both pure marrow cavity and cancellous bone. After fixation, nail 
body is in pure marrow cavity; and screw part is in effective marrow cavity. Thickness of cancellous bone is $1.5 \sim 2 \mathrm{~mm}$, enough to include $1 \sim 1.5 \mathrm{~mm}$ screwing part of distal intramedullary nail. Therefore, when rotating the nail body, screw thread is effective in pressing the fracture site. Additionally, marrow cavity is very slim or disappears at 25/30 of humerus. Therefore, when the thread enters $25 / 30$ of humerus, it is most effective for pressing, and can have a very good control of bone to fix the fracture. So it is very important to select the right length of intramedullary nail beforehand. It would better to compare ample choices of nails under the real-time X-ray with the actual target. Do not compare with the X-ray film because X-ray film usually has distortion, leading to discomfort length, loosened fixation or inadequate pressure, etc., proximal humerus has no bone marrow cavity. Humeral body has $135^{\circ}$ angle with the humeral head [6]. Humeral anatomical neck has $45^{\circ}$ angle with humerus body. In order to make firm locking, we designed a $45^{\circ}$ angle ramp lock structure at the end of nail, making the nail go through the anatomical neck and, therefore, can fix the intramedullary firmly. After the nail is nailed in, the hole for locking nail should be at the same plane of anatomical neck. Therefore, the length of nail also determines its firmness. Intramedullary nail uses distal cancellous bone screw to press in order not to harm the inner cancellous bone. This means rotated forwarding without expanding the cavity. It would maintain the integrity of bone membrane, and is conducive to fracture healing. This additionally requires appropriate length and thickness of intramedullary nail. Thickness should in general be 2-3mm thinner than effective marrow cavity. At least, nail body should not be over pure marrow cavity; and screw thread should not be over effective cavity.

\section{Principles of fixation: SPCLIN has four principles}

(1) Intramedullary running-through effectively prevents lateral and angular displacement of fracture; (2) cancellous bone screw of enlarged nail tail and nail tip forms axial pressure in rotation, leading to tight intercalation of fracture sites. This prevents rotation of fracture sites; (3) proximal locking screw and distal cancellous bone screw can prevent stretching and separation of fractures sites, which can also prevent rotation of fracture sites [7]; (4) humeral neck of proximal humerus is firm compact bone, which can be used by locking nail to nail securely intramedullary nail. Distal $1 / 4$ bone marrow cavity is irregular and surrounded by a lot of trabecular staggered bone structure. This is inner cancellous bone, and can be used for fixation of distal screw thread. Therefore, SPCLIN can fix humeral fractures very well.

\section{Intensity of fixation}

The primary purpose of fixation is to resist against displacement and over-activity of fracture sites and to maintain matching status of fracture fragments to provide the necessary conditions for fracture healing. Traditional Chinese medicine treated middle humerus stable fracture with external splinting by a small plate. It is a widely-acknowledged effective solution to the fixation problem in fracture healing. Compared to wooden splinting, steel intramedullary nail has much greater mechanical strength. Since the application of strong internal fixation, internal fixation is always thought to be better when it is stronger. However, clinical practice has proved that excessive strength leads to stress shielding, bone resorption, even non-healing, or fracture etc. Under normal circumstances, maximum reaction force of the elbow is 10 to 22 times of holding force. When an adult is wearing clothes or having meals, reaction force of elbow is $300 \mathrm{~N}$; and $340 \mathrm{~N}$ for standing up from the chair. But the reaction force is much smaller in the middle of the humerus. From our mechanical test data and analysis, SPCLIN can meet the mechanical requirements of humerus internal fixation.

\section{Fracture healing}

According to recent literature, pressed steel splinting has $75 \%$ excellent healing rate for humerus fractures. Ender nailing has excellent healing rate of $82.6 \%$. Intramedullary nail with locking has $96.4 \%$ excellent healing rate [8]. In our 1006 SPCLIN cases, 755 were healed in eight weeks; six months follow-up showed no non-healing, suggesting a very good therapeutic effect. This might be attributed to the following factors: (1) we don't expand marrow cavity when using the nail; nail body is $1 \mathrm{~mm}$ thinner than pure marrow cavity. Thread part is $1-2 \mathrm{~mm}$ thinner than effective marrow cavity. It does not harm the inner periosteum, maintaining very good conditions for the fracture healing. $\mathrm{Gu}$ et al., hold the view that "intramedullary nails currently used are cylindrical; nails come very close bone pitch, leading to hindered blood circulation and elevated intramedullary pressure [9]. This affects the heart-ward blood coming from the periosteum, and, as a result, is detrimental to the healing of fracture." (2) Through rotating the front screw, the enlarged nail tail can press the fracture sites, which is beneficial to fracture healing; (3) after fixed, SPCLIN doesn't require other supplemental external fixations. So patients can have early shoulder and elbow functional training, which might improve the blood circulation and contribute to fracture healing.

\section{Advantages of SPCLIN}

SPCLIN is simple in structure, and has only intramedullary and locking nails. It is easy to learn, understand and use. Intramedullary nails currently used such as G-K nail, K-S nail, fish mouth interlocking nail, grooved interlocking nail and bifurcated interlocking nail all require open reduction [10]. SPCLIN has sharp tip that can be used for spin entrance. When locking, it doesn't require extra incision, and, therefore, has no need for complicated procedures associated with other nails. In general, a G-K-type intramedullary nailing takes 1.5 to 2 hours to install. However, SPCLIN takes only 20 minutes, greatly saving doctors' labor and patients' pain. Price of SPCLIN is as low as 200-300 Chinese Yen compared with other locking intramedullary nails that are usually priced above 3,000 Chinese Yen (domestic production). In addition, both nailing and removal are percutaneous, largely saving the cost of patients.

\section{Cautions}

Efficacy of SPCLIN has a lot to do with its length. Be sure to choose one with appropriate length and thickness. The nail tail should not be too much over greater tuberosity, so as not to affect shoulder's functional activities. Entrance point should be accurate at greater tuberosity that is close to inter-tuberosity groove and close to center (Figure 6). Do not enter at muscle's ending point, avoiding damage to the muscle. For comminuted fracture of long segment, do not add too much pressure, so as to avoid increased separation, overlapping or displacement of bone fragments. When removing intramedullary nails, be sure to identify the exact location; do not wander around, so as not to damage the shoulder cuff and impact shoulder's function. 


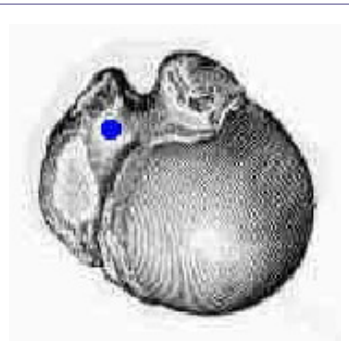

Figure 6: Schematic diagram of entrance point of nailing

\section{References}

1. Williams GR, Ramsey ML, Wiesel SW (2010) Operative Techniques in Shoulder and Elbow Surgery. Lippincott Williams and Wilkins, Philadelphia, USA.

2. Brumback RJ, Bosse MJ, Poka A, Burgess AR (1986) Intramedullary stabilization of humeral shaft fractures in patients with multiple trauma. J Bone Joint Surg Am 68: 960-970.

3. Müller ME, Allgöwer M, Schneider R, Willenegger H (1979) Manual of Internal Fixation, Springer verlag, New York, USA.
4. Richards RR, An KN, Bigliani LU, Friedman RJ, Gartsman GM, et al. (1994) A standardized method for the assessment of shoulder function. J Shoulder Elbow Surg 3: 347-352.

5. Dehong P, Zheng ancestral roots (2000) Anatomical measurement of the humerus bone marrow cavity and the correlation between the humeral interlocking intramedullary nail design. The Orthopedic Journal of China 7: 862-865.

6. Wang Qihua, Sun Bo (1996) Clinical anatomy books. People's Health Publishing House, Beijing, China.

7. Wang Jin, Wang Jielin (1988) Orthopedic biomechanics, 1st edition, People's Health Publishing House, Beijing, China 163.

8. Chen Cai-Ping, Feng Ectocarpus, Yang Dexing (2002) Clinical Efficacy of humeral shaft fracture fixation methods three. Journal of Bone and Joint Surgery 17: 296-297.

9. Gu Long Hall, Jia-wen, Wu Lianghao (2002) Intramedullary nail and plate fixation of long bone fracture analysis. Journal of Bone and Joint Surgery 17: 283-285.

10. Luo is, QIU Gui-xing (1997) Intramedullary nail fixation. People's Health Publishing House, Beijing, China. 